\title{
Effect of bottom roughness on sediment transport due to streaming beneath linear propagating waves with an angle of attack on current
}

\author{
Mohammad Saud Afzal, Lars Erik Holmedal \& Dag Myrhaug \\ Department of Marine Technology \\ Norwegian University of Science and Technology, Trondheim, Norway
}

\begin{abstract}
The effect of bottom roughness on sediment transport due to three-dimensional wave-induced streaming in the seabed boundary layer has been investigated for following and opposing linear propagating waves and current where the wave propagation forms a non-zero angle with the current. Visualizations are given by mean Eulerian wave-averaged suspended flux profiles, as well as the time series of bed shear stress over a wave period. The bedload transport rate along with suspended flux and total sediment transport rate have been presented. For linear propagating waves, the turbulence is induced by the Longuet-Higgins streaming and the classical wave-current interaction. Sediment transport is always in the wave propagation direction for the Longuet-Higgins streaming and increases with decreasing bottom roughness, or as the mean grain diameter decreases.
\end{abstract}

\section{INTRODUCTION}

Wave-current interaction in intermediate and shallow waters is an important phenomenon with regards to ecological settings and engineering applications in the area. This interaction is characterized by turbulent bottom boundary layer near the sea bed which is the driving force for the inception of sediment transport in bottom boundary layers as bedload and/or suspended loads which comprise of chemical compounds, sediments as well as fish larvae and phytoplankton.

Grant \& Madsen (1979) proposed one of the earliest models for combined wave-current bottom boundary layer flow assuming horizontally uniform flow disregarding the small vertical velocity in the bottom boundary layer. This gives a non-zero mass transport and drift in the boundary layer when averaged over a wave period referred to as LonguetHiggins streaming (Longuet-Higgins 1953) who showed that for laminar oscillatory bottom boundary layer flows with sinusoidal forcing, the mass transport is in the same direction as the wave propagation. This result was qualitatively confirmed in measurements by Russel \& Osorio (1958) and Collins (1963) for low Reynolds number (i.e. nearly laminar) flow over a smooth bottom. However, Collins measurements revealed that the steady streaming velocity was reduced in magnitude as the flow became more turbulent. This was confirmed in wave flume experiments for rough bottoms conducted by Bijker et al. (1974) and van Doorn (1981). Another streaming mechanism due to wave asymmetry exists which was investigated, among others, by Davies \& Li (1997) and Scandura (2007). Holmedal et al. (2004) investigated the effect of asymmetric streaming on sediment transport for horizontally uniform flow beneath co-linear waves and current. Other studies such as Holmedal \& Myrhaug (2009) have focused on sediment transport due to the Longuet-Higgins streaming mechanism under waves alone, while Holmedal et al. (2013) have focused on following and opposing waves and current. Recently, Afzal et al. (2015b) investigated the effect of streaming on the wave-current sea bed boundary layer for waves with an angle of attack on the current using numerical simulations. They studied the interaction between the classical wave-current interaction mechanism and the two streaming mechanisms, also affecting the direction and veering of the resulting current, which cannot be measured neither in closed channels nor in large wave flumes. Overall, Afzal et al. (2015b) yields new insight into wave-current seabed boundary layer flow characteristics.

For linear propagating surface waves the LonguetHiggins streaming mechanism exists along with the classical wave-current interaction, yielding sea bed boundary layer drift resulting in net sediment trans- 
port. The classical wave-current interaction is responsible for the reduction of the mean velocity relative to current alone. The Longuet-Higgins streaming mechanism forces the mean flow in the direction of wave propagation which is due to the presence of a vertical wave velocity within the seabed boundary layer. Afzal et al. (2015a) discussed sediment transport in wavecurrent bottom boundary layer under linear propagating waves at an angle with the current. They found that the mean suspended sediment flux and bedload transport decrease as the angle between linear propagating waves and current increases. They also studied the effect of bottom roughness on sediment transport for following waves and current. The present work investigates the effect of bottom roughness on sediment transport due to streaming beneath linear propagating waves with an angle of attack on current using numerical modelling. To demonstrate the effect, the following angles are chosen; $45^{\circ}, 90^{\circ}$ and $135^{\circ}$ in anticlockwise direction.

\section{FORMULATION OF THE MODEL}

The model considers three-dimensional mass transport due to the interaction of waves and current in bottom boundary layers over an infinitely long flat bottom. The horizontal coordinate $x$ is in the direction of the free stream current, the horizontal coordinate $y$ is in the direction perpendicular to the current, whilst the vertical coordinate $\mathrm{z}$ gives the distance from the seabed (see Fig. 1). The bottom is kept fixed at $z=z_{0}=k_{N} / 30$, where $k_{N}$ is the equivalent Nikuradse roughness.

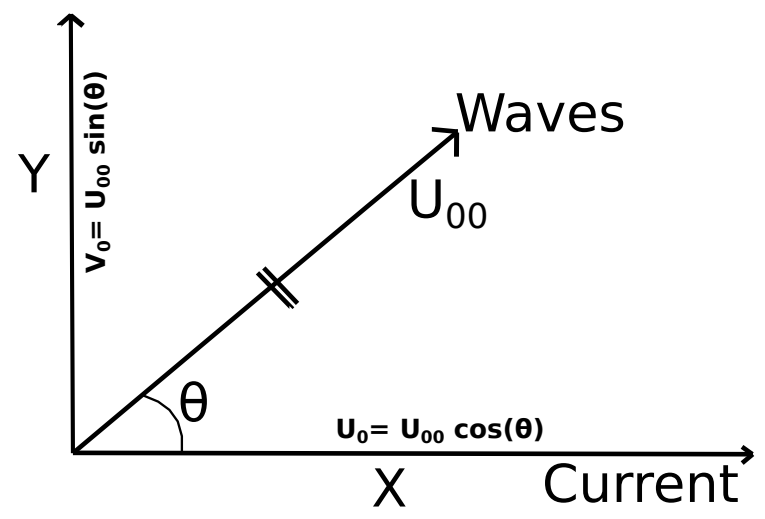

Figure 1: Definition sketch of wave plus current at an angle

The model uses the incompressible unsteady Reynolds-averaged Navier-Stokes (URANS) equations (Eqs. (1) and (2)) and the continuity equation (Eq. (3)) as the governing equations for momentum and mass conservation, respectively.

$\frac{\partial u}{\partial t}+u \frac{\partial u}{\partial x}+v \frac{\partial u}{\partial y}+w \frac{\partial u}{\partial z}=-\frac{1}{\rho} \frac{\partial p}{\partial x}+\frac{\partial}{\partial z}\left(\nu_{T} \frac{\partial u}{\partial z}\right)$

$$
\frac{\partial v}{\partial t}+u \frac{\partial v}{\partial x}+v \frac{\partial v}{\partial y}+w \frac{\partial v}{\partial z}=-\frac{1}{\rho} \frac{\partial p}{\partial y}+\frac{\partial}{\partial z}\left(\nu_{T} \frac{\partial v}{\partial z}\right)
$$

$$
\frac{\partial u}{\partial x}+\frac{\partial v}{\partial y}+\frac{\partial w}{\partial z}=0
$$

where $u$ is the horizontal velocity component in the $\mathrm{x}$-direction, $v$ is the horizontal velocity component in the $y$-direction, $w$ is the vertical velocity component, $p$ is the pressure, $\rho$ is the density of the water, and $\nu_{T}$ is the kinematic eddy viscosity.

The turbulence closure is provided by a $\mathrm{k}-\epsilon$ model which accounts for the bottom roughness. Subject to the bottom boundary layer approximation, this turbulence closure is given as (Rodi 1993);

$$
\begin{aligned}
\frac{\partial k}{\partial t}+u \frac{\partial k}{\partial x}+v \frac{\partial k}{\partial y}+w \frac{\partial k}{\partial z} & =\frac{\partial}{\partial z}\left(\frac{\nu_{T}}{\sigma_{k}} \frac{\partial k}{\partial z}\right) \\
& +\nu_{T}\left(\left(\frac{\partial u}{\partial z}\right)^{2}\right. \\
& \left.+\left(\frac{\partial v}{\partial z}\right)^{2}\right)-\epsilon \\
\frac{\partial \epsilon}{\partial t}+u \frac{\partial \epsilon}{\partial x}+v \frac{\partial \epsilon}{\partial y}+w \frac{\partial \epsilon}{\partial z} & =\frac{\partial}{\partial z}\left(\frac{\nu_{T}}{\sigma_{\epsilon}} \frac{\partial \epsilon}{\partial z}\right) \\
& +c_{\epsilon 1} \frac{\epsilon}{k} \nu_{T}\left(\left(\frac{\partial u}{\partial z}\right)^{2}\right. \\
& -c_{\epsilon 2} \frac{\epsilon^{2}}{k}
\end{aligned}
$$

where $k$ is the turbulent kinetic energy and $\epsilon$ is the turbulent dissipation rate. The kinematic eddy viscosity is given by

$$
\nu_{T}=c_{1} \frac{k^{2}}{\epsilon}
$$

The standard values of the model constants have been adopted, i.e. $\left(c_{1}, c_{\epsilon 1}, c_{\epsilon 2}, \sigma_{k}, \sigma_{\epsilon}\right)=(0.09,1.44$, 1.92, 1.00, 1.30) (Rodi 1993).

The relations $\partial / \partial x=-\cos (\theta)\left(1 / c_{p}\right) \partial / \partial t$ and $\partial / \partial y=-\sin (\theta)\left(1 / c_{p}\right) \partial / \partial t$, where $c_{p}$ is wave celerity and $\theta$ is the angle between the waves and the free stream current, are applied to simplify the momentum and continuity equations. 
The instantaneous dimensionless bedload transport $\Phi$ is a function of the instantaneous dimensionless sea bed shear stress $\Theta$ (Shields parameter) and is given by (Nielsen 1992)

$\Phi=12 \Theta^{\frac{1}{2}}\left(\Theta-\Theta_{c}\right) \frac{\Theta}{|\Theta|}$

where

$$
\begin{aligned}
\Phi & =\frac{q_{b}}{\left(g(s-1) d_{50}^{3}\right)^{\frac{1}{2}}} \\
\Theta & =\frac{\tau_{\mathrm{b}}}{\rho g(s-1) d_{50}}
\end{aligned}
$$

Here $q_{b}$ is the instantaneous dimensional bedload transport, $\tau_{\mathrm{b}}$ is the dimensional instantaneous sea bed shear stress, $g$ is the gravity acceleration, $s=2.65$ is the density ratio between the bottom sediments and the water, and $d_{50}$ is the median grain size diameter. The critical Shields parameter $\Theta_{c}=0.05$ must be exceeded for bedload transport to take place.

By using the boundary layer approximation, the equation for the sediment concentration $c$ is:

$$
\begin{aligned}
\frac{\partial c}{\partial t}+u \frac{\partial c}{\partial x}+v \frac{\partial c}{\partial y}+\left(w-\mathrm{w}_{s}\right) \frac{\partial c}{\partial z} & =\frac{\partial}{\partial z}\left(\epsilon_{s} \frac{\partial c}{\partial z}() 10\right) \\
\epsilon_{s} & =\nu_{T}+\nu
\end{aligned}
$$

Here $\epsilon_{s}$ is the sediment diffusivity, $\mathrm{w}_{s}$ is the settling velocity of the median sand grains in still water, and $\nu$ is the kinematic viscosity of water. Here the kinematic viscosity has been included in the sediment diffusivity in order to stabilize the numerical scheme; this model is described in more detail in Holmedal et al. (2004).

At the bed level $z_{0}=k_{N} / 30$ the no-slip condition (Eq. (12)) is applied since the sea bed is assumed to be hydraulically rough.

$u=0, v=0, w=0$ at $z=z_{0}$

For the turbulent quantities near the bed (k and $\epsilon$ ), the boundary conditions are given in a standard manner (Rodi 1993). By assuming local equilibrium between production and dissipation, the boundary conditions at $z=z_{0}$ become

$$
\begin{aligned}
k & \left.=\nu_{T}\left(\left(\frac{\partial u}{\partial z}\right)^{2}+\left(\frac{\partial v}{\partial z}\right)^{2}\right)\right)^{1 / 2} / \sqrt{c_{1}} \\
\epsilon & =\left(c_{1}\right)^{\frac{3}{4}} \frac{k^{\frac{3}{2}}}{\kappa z_{0}}
\end{aligned}
$$

where the flow is assumed to be parallel to the wall in the close vicinity of the bottom, i.e. on the computational node nearest the bottom. Here $\kappa=0.4$ is the von Karman constant.

The velocity for waves plus current at $z=z_{\max }$, is given as:

$u=U_{00} \cos \theta+U_{c}, v=U_{00} \sin \theta$

where $U_{00}$ is the harmonic component of the velocity (see Fig. 1), $U_{c}$ is the mean current velocity, and $\theta$ is the angle of attack of waves on current at the outer boundary layer $z_{\max }$. Zero flux conditions of the turbulent quantities $\mathrm{k}$ and $\epsilon$ are imposed at $z=z_{\max }$, giving

$$
\begin{aligned}
& \frac{\partial k}{\partial z}=0 \\
& \frac{\partial \epsilon}{\partial z}=0
\end{aligned}
$$

The applicability of the zero flux conditions of the turbulent quantities to waves plus current is discussed in Holmedal et al. (2003); it appears that these conditions yield a realistic representation of the seabed boundary layer near the seabed, which is of primary interest to this investigation.

The reference sediment concentration $c_{a}$ near the sea bed is obtained from the instantaneous Shields parameter $\Theta$ by the Zyserman \& Fredsøe (1994) formula. A zero flux condition (Eq. (18)) is also imposed on the sediment concentration at at $z=z_{\max }$.

$$
\nu_{T} \frac{\partial c}{\partial z}+\mathrm{w}_{s} c=0
$$

\section{RESULTS AND DISCUSSION}

The effect of bottom roughness on sediment transport within the ocean wave-current bottom boundary layer for realistic wave conditions and water depth is presented. Ocean surface waves with an amplitude of $a=1.22 \mathrm{~m}$ and a period of $6 \mathrm{~s}$ propagate over a flat rough bottom, with a current specified as $U_{c}=0.1 \mathrm{~m} / \mathrm{s}$ at $z_{\max }=0.25 \mathrm{~m}$ above the bottom. The angle $\theta$ between current and waves are $45^{\circ}, 90^{\circ}$ and $135^{\circ}$, the water depth is $8 \mathrm{~m}$ and the resulting wave length is $45 \mathrm{~m}$. The given wave conditions represent intermediate water depth $\left(k_{p} h=1.11\right)$ with wave steepness $a k_{p}=0.17$, where $k_{p}$ represents wave number. The median sand grain diameters are $d_{50}$ $=0.13 \mathrm{~mm}, 0.21 \mathrm{~mm}$ and $0.32 \mathrm{~mm}$, corresponding to $\mathrm{A} / k_{\mathrm{N}}=2900,1800$ and 1180 , respectively, where $\mathrm{A}$ is the near-bottom wave excursion amplitude and 


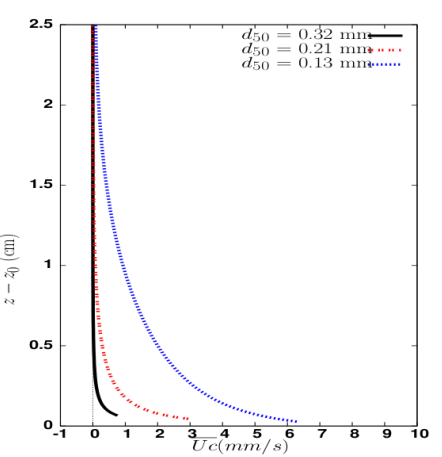

(a)

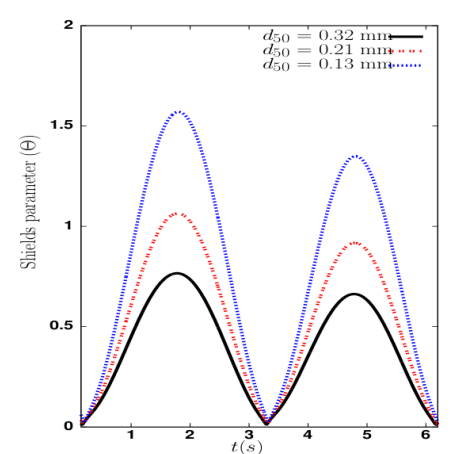

(b)
Figure 2: a) Sediment flux b) magnitude of the Shields parameter for linear propagating waves at an angle of $45^{\circ}$ with the current for three different sediment sizes

$k_{\mathrm{N}}=2.5 d_{50}$. The corresponding settling velocities are $w_{s}=0.0119 \mathrm{~m} / \mathrm{s}, 0.026 \mathrm{~m} / \mathrm{s}$ and $0.0429 \mathrm{~m} / \mathrm{s}$.

Fig. 2 shows the Eulerian wave-averaged suspended sediment flux profiles (Fig. 2 (a)) and the magnitude of the Shields parameter over one wave cycle (Fig. 2 (b)) for three median grain diameters $\left(d_{50}=0.13 \mathrm{~mm}, 0.21 \mathrm{~mm}\right.$ and $\left.0.32 \mathrm{~mm}\right)$ for linear propagating waves at angle of $45^{\circ}$ with the current for $T_{p}=6 \mathrm{~s}, U_{c}=0.1 \mathrm{~m} / \mathrm{s}$. It should be noted that the increasing median grain diameter corresponds to increasing bottom roughness. From Fig. 2 (a) it appears that the mean sediment flux increases as $d_{50}$ decreases, as expected. This observation is also supported by the decrease of the Shields parameter as $d_{50}$ increases. For linear propagating waves the magnitude of the Shields parameter is larger beneath the wave crest than beneath the wave trough as can be seen in Fig. 2 (b). This is because the LonguetHiggins streaming induces a wave-average bed shear stress in addition to the wave-averaged bed shear stress due to the current in the wave propagation direction for all angles between the waves and the current.

Table 1: Mean bedload transport, suspended sediment transport and total transport $\left(\bar{q}_{\text {total }}\right)$ for three different median grain diameters beneath linear propagating waves at an angle of $45^{\circ}$ with the current. Here $U_{c}=0.1 \mathrm{~m} / \mathrm{s}, T_{p}=6 \mathrm{~s}$

\begin{tabular}{cccccc}
\hline$d_{50}$ & $\bar{q}_{b x}$ & $\bar{q}_{b y}$ & $\bar{q}_{b t}$ & $\int_{2 d_{50}}^{z_{\max }} \overline{U c} d z$ & $\bar{q}_{\text {total }}$ \\
$m m$ & $\mathrm{~mm}^{2} / \mathrm{s}$ & $\mathrm{mm}^{2} / \mathrm{s}$ & $\mathrm{mm}^{2} / \mathrm{s}$ & $\mathrm{mm}^{2} / \mathrm{s}$ & $\mathrm{mm}^{2} / \mathrm{s}$ \\
\hline 0.13 & 7.00 & 5.30 & 8.79 & 26.37 & 35.16 \\
0.21 & 7.79 & 6.03 & 9.86 & 4.58 & 14.44 \\
0.32 & 8.71 & 6.86 & 11.08 & 0.66 & 11.74 \\
\hline
\end{tabular}

Table 1 shows mean bedload transport rate, suspended sediment transport rate and total transport rate for the three different median grain diameters beneath linear propagating waves at an angle of $45^{\circ}$ with the current, where $\bar{q}_{b x}$ and $\bar{q}_{b y}$ are the x- and $\mathrm{y}$-components, respectively, of the mean bedload transport rate $\bar{q}_{b t}, \int_{2 d_{50}}^{z_{\max }} \overline{U c} d z$ is the mean suspended sediment transport rate, and $\bar{q}_{\text {total }}$ is the total sediment transport rate (the mean suspended sediment transport plus the total mean bedload transport). Here, $U$ represents the total velocity vector. The results in Table 1 show that the bedload transport increases slightly as $d_{50}$ increases. This is expected as the total bedload transport rate depends upon both the Shields parameter and $d_{50}$, which have competing effects on the total bedload transport (see Eq. (9)). Also the formulation used for calculation of bedload in the numerical model is purely empirical in nature and does not account for features like packing density and void ratio. However, the suspended sediment flux and the total sediment transport rate decreases as $d_{50}$ increases, as expected.

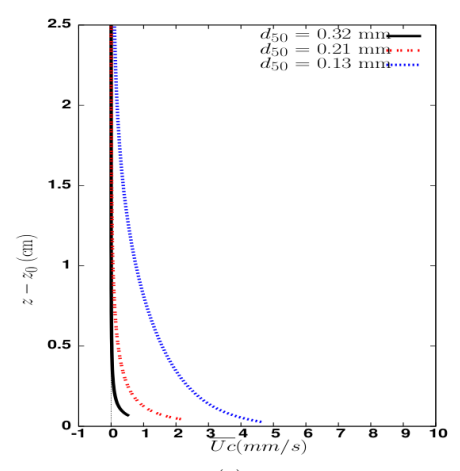

(a)

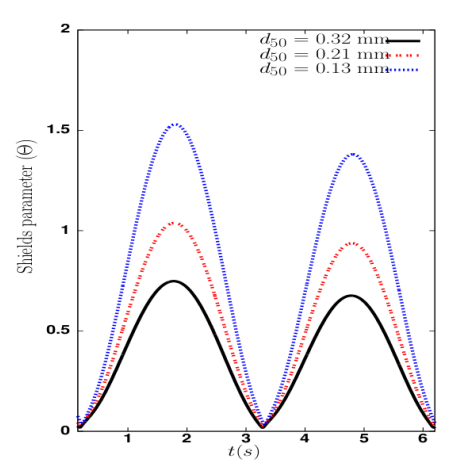

(b)
Figure 3: a) Sediment flux b) magnitude of the Shields parameter for linear propagating waves at an angle of $90^{\circ}$ with the current for three different sediment sizes

Table 2: Mean bedload transport, suspended sediment transport and total transport $\left(\bar{q}_{\text {total }}\right)$ for three different median grain diameters beneath linear propagating waves at an angle of $90^{\circ}$ with the current. Here $U_{c}=0.1 \mathrm{~m} / \mathrm{s}, T_{p}=6 \mathrm{~s}$

\begin{tabular}{cccccc}
\hline$d_{50}$ & $\bar{q}_{b x}$ & $\bar{q}_{b y}$ & $\bar{q}_{b t}$ & $\int_{2 d_{50}}^{z_{\max }} \overline{U c} d z$ & $\bar{q}_{\text {total }}$ \\
$m m$ & $m m^{2} / s$ & $m m^{2} / s$ & $\mathrm{~mm}^{2} / s$ & $\mathrm{~mm}^{2} / s$ & $\mathrm{~mm}^{2} / \mathrm{s}$ \\
\hline 0.13 & 1.87 & 6.14 & 6.42 & 21.44 & 27.86 \\
0.21 & 1.97 & 7.03 & 7.31 & 3.38 & 10.69 \\
0.32 & 2.08 & 8.04 & 8.31 & 0.48 & 8.79 \\
\hline
\end{tabular}

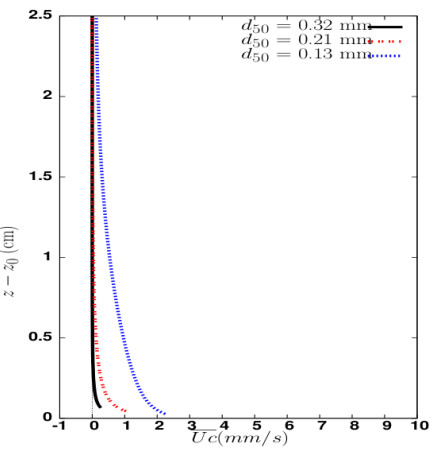

(a)

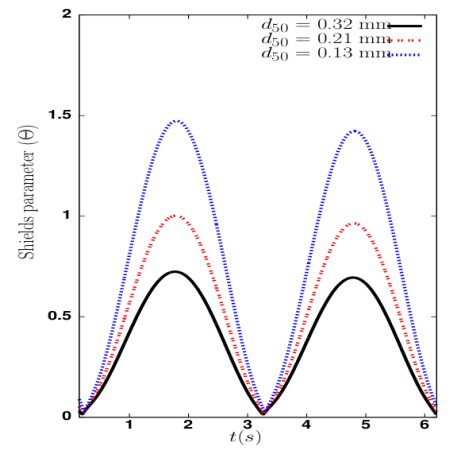

(b)
Figure 4: a) Sediment flux b) magnitude of the Shields parameter for linear propagating waves at an angle of $135^{\circ}$ with the current for three different sediment sizes 
Table 3: Mean bedload transport, suspended sediment transport and total transport $\left(\bar{q}_{\text {total }}\right)$ for three different median grain diameters beneath linear propagating waves at an angle of $135^{\circ}$ with the current. Here $U_{c}=0.1 \mathrm{~m} / \mathrm{s}, T_{p}=6 \mathrm{~s}$

\begin{tabular}{cccccc}
\hline$d_{50}$ & $\bar{q}_{b x}$ & $\bar{q}_{b y}$ & $\bar{q}_{b t}$ & $\int_{2 d_{50}}^{z_{\max }} \overline{U c} d z$ & $\bar{q}_{\text {total }}$ \\
$m m$ & $\mathrm{~mm}^{2} / \mathrm{s}$ & $\mathrm{mm}^{2} / \mathrm{s}$ & $\mathrm{mm}^{2} / \mathrm{s}$ & $\mathrm{mm}^{2} / \mathrm{s}$ & $\mathrm{mm}^{2} / \mathrm{s}$ \\
\hline 0.13 & -0.80 & 2.96 & 3.06 & 14.29 & 17.35 \\
0.21 & -1.2 & 3.46 & 3.67 & 1.75 & 5.42 \\
0.32 & -1.6 & 4.02 & 4.34 & 0.24 & 4.58 \\
\hline
\end{tabular}

Figs. 3 and 4 show the Eulerian wave-averaged suspended sediment flux profiles and the Shields parameter over one wave cycle for three median grain diameters $\left(d_{50}=0.13 \mathrm{~mm}, 0.21 \mathrm{~mm}\right.$ and $\left.0.32 \mathrm{~mm}\right)$ for $T_{p}$ $=6 \mathrm{~s}, U_{c}=0.1 \mathrm{~m} / \mathrm{s}$ for linear propagating waves at an angle of $90^{\circ}$ and $135^{\circ}$, respectively, with the current, whereas Tables 2 and 3 show the corresponding mean bedload transport, suspended sediment transport and total transport. These results indicate that with increasing bottom roughness (mean grain diameter), the suspended sediment flux decreases for all the angles between the waves and the current. The magnitude of the Shields parameter is larger beneath the wave crest than beneath the wave trough (Figs. 3 (b) and 4 (b)) due to the extra bedshear stress induced in the direction of wave propagation due to the Longuet-Higgins streaming. The results with $\theta=90^{\circ}$ and $135^{\circ}$ (shown in Figs. 3 and 4, respectively) are qualitatively similar to those for $\theta=45^{\circ}$ (shown in Fig. 2). It can also be seen from Figs. 2, 3 and 4 that both the suspended sediment flux and the magnitude of the Shields parameter decreases as the angle between the waves and the current increases. The results shown in Tables 2 and 3 are also qualitatively similar to that of Table 1 indicating that the bedload transport rate increases slightly as the bottom roughness increases, whereas the total sediment transport rate decreases with increasing bottom roughness. It should also be noted that the bedload transport rate, suspended flux and the total sediment transport rate decreases with increasing angle between the waves and the current.

\section{CONCLUSIONS}

Sediment transport in the wave-current bottom boundary layer under linear propagating waves at an angle with the current has been investigated for different bottom roughnesses (sediment grain sizes). The results obtained using these conditions should be qualitatively similar to those obtained for other wave-dominated wave-current flows where the effect of streaming is important. The main results are as follows:

- The suspended load decreases with increasing bottom roughness.

- The total sediment transport decreases with increasing bottom roughness.
- The bedload rate increases slightly with increasing bottom roughness.

- The mean suspended sediment flux and bedload transport decreases as the angle between the linear propagating waves and current increases.

\section{ACKNOWLEDGMENTS}

This work was carried out as part of the strategical university program "Air-Sea Interaction and Transport Mechanisms in the Ocean", funded by the Norwegian Research Council.

\section{REFERENCES}

Afzal, M. S., L. E. Holmedal, \& D. Myrhaug (2015a). Sediment transport in wave-current bottom boundary layer under linear propagating waves at an angle with the current, Chapter 93. World Scientific.

Afzal, M. S., L. E. Holmedal, \& D. Myrhaug (2015b). Threedimensional streaming in the seabed boundary layer beneath propagating waves with an angle of attack on the current. Journal of Geophysical Research: Oceans, n/a-n/a.

Bijker, E. W., J. P. T. Kalkwijk, \& T. Pieters (1974, June). Mass transport in gravity waves on a sloping bottom. In 14'th International Conference on Coastal Eng., Copenhagen, pp. 2428.

Collins, J. I. (1963). Inception of turbulence at the bed under periodic gravity waves. J. Geophys. Res. 68, 6007-6014.

Davies, A. G. \& Z. Li (1997). Modelling sediment transport beneath regular symmetrical and asymmetrical waves above a plane bed. Continental Shelf Research 17(5), 555-582.

Grant, W. D. \& O. S. Madsen (1979). Combined wave and current interaction with a rough bottom. Journal of Geophysical Research: Oceans 84(C4), 1797-1808.

Holmedal, L. E., J. Johari, \& D. Myrhaug (2013). The seabed boundary layer beneath waves opposing and following a current. Continental Shelf Research 65(0), 27 - 44.

Holmedal, L. E. \& D. Myrhaug (2009). Wave-induced steady streaming, mass transport and net sediment transport in rough turbulent ocean bottom boundary layers. Continental Shelf Research 29(7), $911-926$.

Holmedal, L. E., D. Myrhaug, \& K. J. Eidsvik (2004). Sediment suspension under sheet flow conditions beneath random waves plus current. Cont. Shelf Res. 24, 2065-2091.

Holmedal, L. E., D. Myrhaug, \& H. Rue (2003). The sea bed boundary layer under random waves plus current. Cont. Shelf Res. 23(7), 717-750. erratum 1035.

Longuet-Higgins, M. S. (1953). Mass transport in water waves. Philosophical Transactions of the Royal Society of London. Series A, Mathematical and Physical Sciences 245(903), pp. 535-581.

Nielsen, P. (1992). Coastal bottom boundary layers and sediment transport, Volume 4. World scientific.

Rodi, W. (1993). Turbulence Models and Their Application in Hydraulics, A state-of-the-art review. (third ed.). IAHR Monograph series. A. A. Balkema, Rotterdam, Netherlands.

Russel, R. C. H. \& J. D. C. Osorio (1958). An experimental investigation of drift profiles in a closed channel. In Proc. 6th Int. Conf. Coastal Eng., Miami, pp. 171-193. ASCE.

Scandura, P. (2007). Steady streaming in a turbulent oscillating boundary layer. Journal of Fluid Mechanics 571, 265-280.

Trowbridge, J. \& O. S. Madsen (1984). Turbulent wave boundary layers: 2. Second-order theory and mass transport. Journal of Geophysical Research: Oceans 89(C5), 7999-8007. 
van Doorn, T. (1981). Experimental investigation of near-bottom velocities in water waves without and with a current. Report of investigations M1., Delft Hydraulics Laboratory.

Zyserman, J. A. \& J. Fredsøe (1994). Data analysis of bed concentration of suspended sediment. Journal of Hydraulic Engineering 120(9), 1021-1042. 